\title{
CNT-based RFID Passive Gas Sensor
}

\author{
Cecilia Occhiuzzi*, Amin Rida ${ }^{\diamond}$, Gaetano Marrocco*, Manos M. Tentzeris ${ }^{\diamond}$ \\ *DISP - University of Roma Tor Vergata \\ Via del Politecnico, 1, 00133, Roma (ITALY) \\ $\diamond_{E C E}$ - Georgia Institute of Technology \\ 85, 5th Street NW, Atlanta, GA 30308, (US)
}

\begin{abstract}
In this paper Single-Wall Carbon Nanotube (CNT) are examined for the design of a passive and maintenance-free wireless RFID sensor. CNT buckypaper is characterized from a dielectric and a sensititvity point of view, using an indirect way, by using antenna measurement and simulations in a controlled medium. A CNT-based prototype RFID tag is then described as the featured maintenance free sensor and experimentally verified for its applicability and sensitivity towards $\mathrm{NH}_{3}$.
\end{abstract}

\section{INTRODUCTION}

There has been a vast movement towards combining the capabilities of a sensor and an RF or wireless device in order to sense, process data, and transmit information about objects/personnel. Radio Frequency Identification (RFID) passive sensing has been successfully explored as a low cost candidate or solution towards lightweight, reliable, energy efficient and even battery less durable wireless sensor devices. In addition to RFID's basic identification capabilities, the integration of sensors finds existing and novel applications such as temperature sensing, smart skin applications or structural health monitoring, bio-sensing, and gas sensing. Therefore there is a demand for RFID-enabled sensors, and many challenges remain to overcome. Existing examples of RFID-enabled sensors include: temperature sensing, solar powered and piezoscavenging battery less wireless sensor tags, human body monitoring by means of implanted RFID tags, passive RFID for biological detection, passive RFID based strain sensors, wearable passive RFID motion sensor, remote moisture sensing, and Gas-Detection.

While the sensing applications list grows, a major interest lies in the remote monitoring of environments such as sensing the air for the presence of hazardous gases in industrial environments like supply chain and food monitoring or for health monitoring of workers. Traditional gas sensing mechanisms are made using semi-conducting oxides as the sensing material which require micro-fabrication techniques, power supply and ad-hoc electronics. Carbon Nanotubes (CNT) composites [1] have been found to perform as a gas sensor by possessing electrical conductance highly sensitive to small quantities of ammonia $\left(\mathrm{NH}_{3}\right)$ and other gaseous [2]. It is not surprising either that $\mathrm{CNT}$ and other nanoscale devices are being thoroughly studied for a wide range of applications for high frequency electronics including chemical sensors, field effect transistors, and nanoelectromechanical systems [3]. Several CNT based antennas have also been developed for their attractive characteristics. In [4] a CNT nanoantenna is stated to be a usually an electrically extremely short antenna, that is considerably shorter than the free-space wavelength due to its very high inductance per unit length owing to its high kinetic energy of the electrons. In [5] the use of carbon fiber composites and CNT composites is investigated for use in RFID antennas and are regarded as low cost, low weight, easy to fabricate, in addition they possess good corrosion resistance as compared to metallic surfaces, better bandwidth and may act as a matching mechanism. In [6] CNT is used to realize conformal antennas on polymer substrates. However in [4], [5], [6] no attempt for using CNT as a sensor was taken.

In this work, the integration of single wall carbon nanotube (SWCNT) with a conventional passive RFID antenna including an IC, is demonstrated as a maintenance-free (or battery-less) wireless ammonia sensor. The SWCNT, after their formation using the buckypaper technique, have been characterized at RF. Then an RFID passive tag was designed and matched to a commercial RFID IC. An inductive coupling method was used where a CNT loading was utilized as a variable load sensitive to the environmental conditions. The feasibility of passive RFID tag acting as sensors is here fully investigated in the American and European UHF band. Different sensor identifiers have been considered for the $\mathrm{NH}_{3}$ gas characterization, showing the successful operation of the passive integrated CNT RFID antenna as a gas sensor.

\section{SWCNT MATERIAL PREPARATION AND CHARACTERIZATION}

Several deposition techniques have been proposed for CNTbased antenna and electronics: inkjet and screen-printing procedures [7], CVD processes [6] and thin films [5] are recent examples of useful techniques able to offer high conductive CNT samples.

SWCNT film, also referred to as buckypaper, can be made by filtering SWCNT dispersion in aqueous or organic media, such as Triton X-100 or dimethyl formamide DMF, under positive or negative pressure. It offers isotropic conductivity, good mechanical strength and flexibility and gives the possibility to easily cut out the shape of the desired antenna.

In this work an alternative preparation method is presented, where the filtration over membrane has been replaced by evaporation in a controlled environment. The process is schematically shown in Fig.1. A $100 \mathrm{mg}$ purified, high functionality with carbonaceous purity $\geq 90 \%$ SWCNT powder from Carbon Solutions, Inc has been dispersed in $66 \mathrm{~mL}$ of 
water by sonication at $30 \mathrm{~W}$ for $60 \mathrm{~min}$. Next, in order to get rid of the water and form the CNT bucky paper, an overnight evaporation of the water at $70^{\circ} \mathrm{C}$ took place. Finally a circular sheet with $r=90 \mathrm{~mm}$ and predicted thickness $t=32 \mu \mathrm{m}$ has been produced over a polyamide membrane. The thickness of the buckypaper is determined by the total amount of CNTs used in the dispersion per unit surface of the membrane. With a calculated density of $1.6 \times 10^{-2} \mathrm{mg} / \mathrm{mm}^{2}$, the thickness is estimated to be $32 \mu \mathrm{m}$ [8].

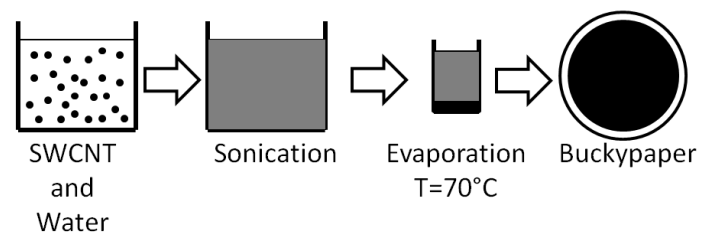

Figure 1. Buckypaper preparation steps.

The sonication and the evaporation/filtration procedures are extremely important for optimizing the CNT buckypaper performance. It is to be noted that large CNT agglomerates in the CNT solution will dominate leading to a brittle sheet, as well as a non uniform evaporation produces non uniform sheet with holes and consequently non isotropic conductive performances. Fig. 2 shows the result of the process: the CNT buckypaper appears macroscopically uniform (a), but some undesired inconsistencies or brittleness towards are present at the center of the sample leading to a non good and uniform conductivity and mechanical properties (b).

The dielectric properties of CNT are strictly dependent on their deposition techniques. Concentration, orientation, number of layers and material type are important parameters that effect the performances of the nanotubes, especially at RF. Although several characterization have been proposed, especially in DC, the dielectric properties are not univocally defined, making necessary to preliminarily produce an RF characterization of the specific produced buckypaper. Here, an indirect characterization method has been used to refine the different values available in literature according to our specific sample. Based on the assumption that CNT is considered as a lossy metal (with $\varepsilon_{r}=1$ and finite conductivity $\sigma$ ), several types of monopole-antennas considering CNT buckypaper as conductive element have been designed, simulated and measured by means of a Vector Network Analyzer (VNA

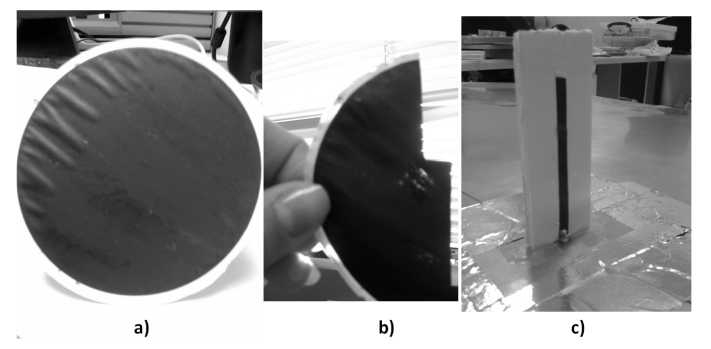

Figure 2. CNT Buckypaper a) prepared optimum sample b) non-optimal sample and c) CNT buckypaper monopole
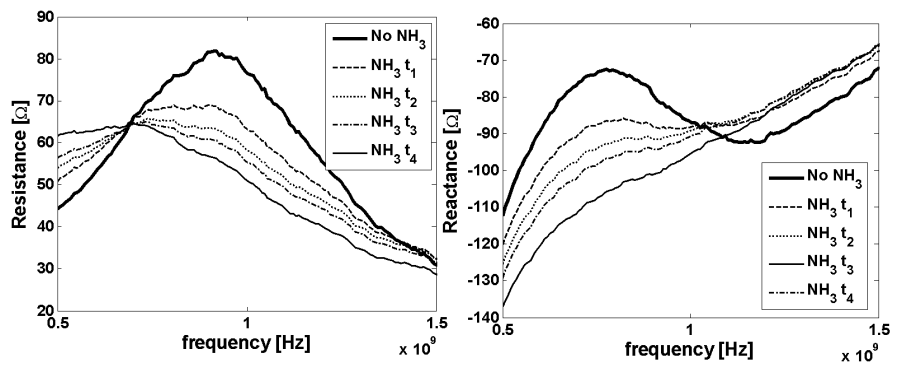

Figure 3. $\mathrm{Z}_{11}$ results of the monopole CNT antenna of Fig. 2 with and without the presence of the $\mathrm{NH}_{3}$.

Rohde\&Schwarz ZVA8). An r.m.s. minimization has been applied in order to fit the measured and simulated input impedances and thus evaluate the conductivity. For each run, the best minimizing $\sigma$ has been considered. After averaging the different results the conductivity of buckypaper has been evaluated as $\sim 2.5 S / m$. The accuracy of these values has been finally verified by designing and testing another monopole antenna.

A strip of the CNT buckypaper of dimensions $80 \mathrm{~mm} x 5 \mathrm{~mm}$ was cut out of the membrane and plated on the substrate in order to form a monopole antenna somewhere in the UHF frequencies. Fig. 2 c) shows the photograph of the monopole antenna attached to a foam backing for support and as was used in the measurement setup. A silver epoxy mixture was applied at the interface CNT antenna - SMA connector and cured for their conductive and mechanical connectivity.

Fig.3 (thick line) shows the measured $\mathrm{Z}_{\text {in }}$ of the monopole CNT antenna. As shown, the monopole is strongly capacitive with a $-110 \leq \mathrm{jX}_{\text {in }} \leq-70$ Ohms and with a resistance $30 \leq \mathrm{R}_{\text {in }} \leq 80$ Ohms. Despite the seemingly low conductivity, the CNT monopole structure acts like an antenna with a measurable $\mathrm{Z}_{i n}$ that is sensitive to the environment. The DC resistance was measured by a multimeter from top of the monopole to the output of the SMA connector with a reading of 278 Ohms.

The next step in the process of characterization was the addition of $\mathrm{NH}_{3}$. A volume of $6 \mathrm{ml}$ of a commercial off the shelf $10 \%$ ammonia hydroxide was guided in a $460 \mathrm{~cm}^{3}$ plastic chamber surrounding the monopole. Upon the addition of the ammonia, a quick and strong response was recorded. This is shown in Fig. 3 where $t 1$ implies the time the measurements were recorded right after the introduction of ammonia. A linear effect is also observed on $\mathrm{R}_{i n}$ and $\mathrm{X}_{i n}$ as time progresses. Here $\mathrm{t} 5$ - $\mathrm{t} 1 \approx 15 \mathrm{mins}$. As time progresses slight saturation in the recorded measurements are observed. While the exact concentration of ammonia ( $p p m)$, is unknown, the proof of concept of using CNT antenna as a chemical sensor is demonstrated.

The $\mathrm{Z}_{i n}$ recovery features are shown in 4 . A linear effect is shown here as well, where $t 1$ implies the time the measurements were recorded right after the removal of ammonia, with recovery times recorded, $\mathrm{t} 4-\mathrm{t} 1 \approx 1$ hour. A slow response time is noted, and a slight hysteresis effect as well, a behavior that 

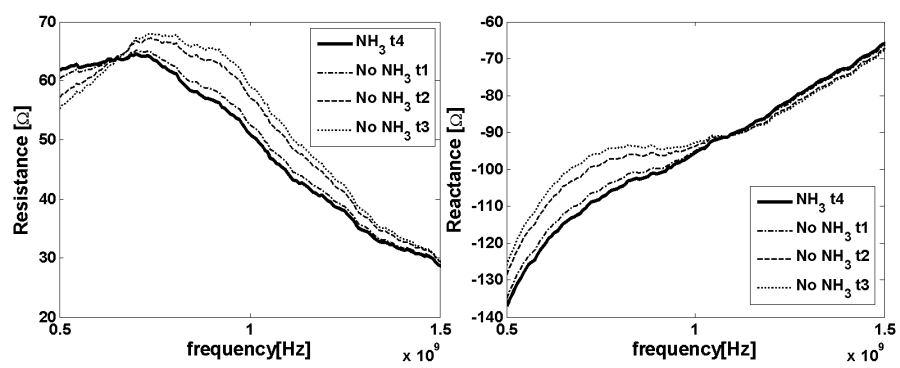

Figure 4. $\mathrm{Z}_{11}$ recovery features of the monopole CNT antenna of Fig. 2 after ammonia removal.

was also noted by [9].

Another observation here is that $\mathrm{NH}_{3}$ produces a decrease in conductivity [9], [7], a phenomenon that was also verified in our measurements/ext actions.

\section{RFID GAS SENSOR:DESIGN AND PROTOTYPE}

There are several options to achieve a gas-sensitive tag taking advantage of the sensing feature of the CNT. A first classification could be done considering the CNT as a part of the antenna radiating structures, e.g. an antenna totally or partially made of nanotube, or considering the buckypaper as a "passive" component of the tag, e.g. a variable load placed in close proximity of the radiating structure. Both solutions offer advantages and drawbacks, the best trade off between communication and sensing must be considered. Considering CNT as a radiating element it is possible to increase the sensitivity of the device, however lowering the antenna efficiency and the activation distance. These two parameters, together with the cost, are essential in RFID systems. The other solution, on the contrary, offers better communication and cost performances but could suffer of weak gas sensitivity.

In this work CNT is used as a loading on a conventional RFID passive tag. The design choice of the RFID tag was an inductively coupled loop [13] RFID tag, designed to match an NXP TSSOP8 RFID IC (www.nxp.com) with complex impedance $\mathrm{Z}_{I C}=16-\mathrm{j} 148$ Ohms. In this design, shown in Fig. 5, the strength of the coupling is controlled by the distance between the loop and the radiating body, as well as by the shape factor of the loop. A rectangular piece of CNT buckypaper, whose conductivity $\sigma$ has been considered equal to $2 \mathrm{~S} / \mathrm{m}$ according to the previous analysis, was then placed in the space between the loop and radiating body. The dimension of the RFID Antenna with the CNT loading are shown in Table I.

A photo of the prototype as well as the setup used for its characterization is shown in Fig. 6. A Voyantic Tagformance (www.voyantic.com) was used for these measurements with a Kushcraft antenna having a $6 \mathrm{dBi}$ gain and a circular polarization. The reader-tag distance is fixed to $63.5 \mathrm{~cm}$. Both the reader and the tag are $101 \mathrm{~cm}$ high from the floor. All the measurements have been conducted into the anechoic chamber.

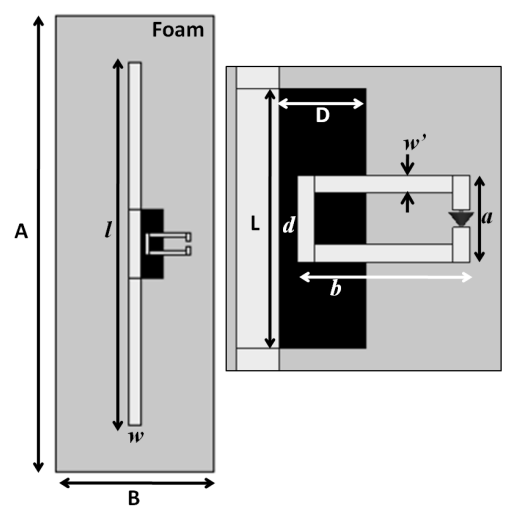

Figure 5. RFID Tag: Inductively Coupled Loop with rectangular CNT loading.

Table I

RFID ANTENNA DIMENSIONS

\begin{tabular}{|c|c|}
\hline Parameter & Dimensions in [mm] \\
\hline$A$ & 180 \\
$B$ & 80 \\
$l$ & 160 \\
$w$ & 5 \\
$L$ & 30 \\
$w^{\prime}$ & 2 \\
$a$ & 10 \\
$b$ & 22 \\
$D$ & 10 \\
\hline
\end{tabular}

\section{Sensing Characterization}

Using the setup in Fig. 6 the sensing performances of the tag have been tested. The tag has been placed into a $684 \mathrm{~cm}^{3}$ plastic gas chamber, and a volume of $6 \mathrm{ml}$ of a commercial off the shelf $10 \%$ ammonia hydroxide was guided into it. A new reference condition for the tag, e.g without ammonia, has been defined considering the presence of the gas chamber in close proximity of the radiating structures. The plots for turn on power $P^{t o}[14]$, was generated in order to officially treat this RFID Tag as a chemical sensor.

Fig. 7 shows the results of the $P^{t o}$ in $d B m$ for the DUT with and without $\mathrm{NH}_{3}$. An immediate and strong response is observed at $\mathrm{t} 1=1$ min followed by a saturated response of $P^{t o}$ at
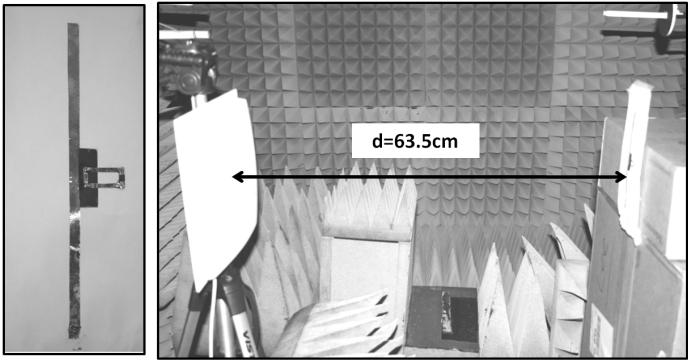

Figure 6. Photo of the Prototype and Setup for testing. 


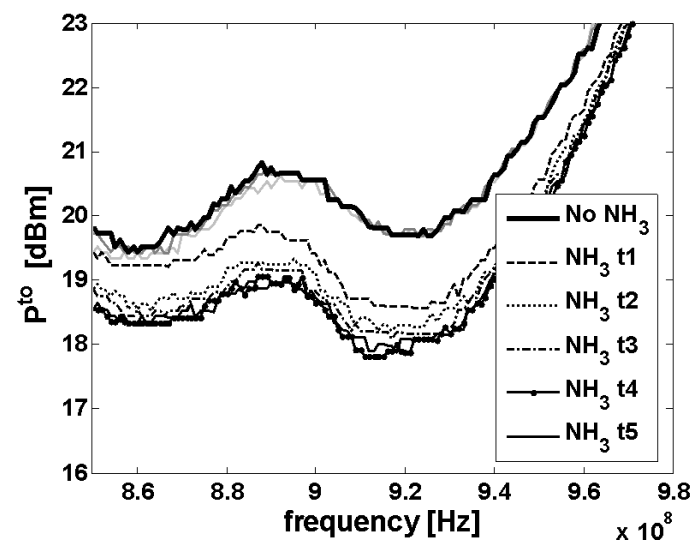

Figure 7. Turn on Power of the CNT loaded RFID Tag with $\mathrm{NH}_{3}$.

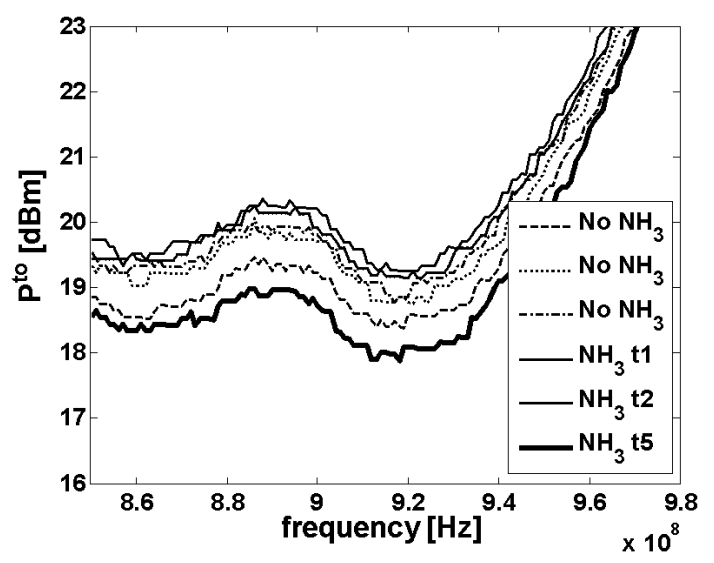

Figure 8. Turn on Power of the CNT loaded RFID Tag - Recovery.

$t 2=4 \mathrm{~min}, t 3=6 \mathrm{~min}, t 4=9 \mathrm{~min}$, and $t 5=15 \mathrm{~min}$. It is also to be noted that several measurements were reported for the no $\mathrm{NH}_{3}$ case with perfectly overlapping $P^{t o}$ curves. Fig. 8 shows the recovery plots of $\mathrm{P}^{t o}$ vs. frequency having removed the ammonia for $t 1=5 \mathrm{~min}, t 2=13 \mathrm{~min}, t 3=40 \mathrm{~min}, t 4=$ $50 \mathrm{~min}$, and $t 6=60 \mathrm{~min}$. A linear recovery characteristic has also been shown here, with a slower response. This proves the proof of concept of a CNT-based RFID passive gas sensor.

\section{CONCLUSIONS}

CNT buckypaper has been realized and indirect characterization methods have been utilized for its characterization. Tests have been conducted to show proof of concept of using the CNT as an $\mathrm{NH}_{3}$ sensor, and finally a novel CNTbased passive RFID Tag functioning as an $\mathrm{NH}_{3}$ sensor was demonstrated as a low cost and maintenance free gas sensor.

\section{REFERENCES}

[1] P. Avouris, J. Appenzeller, R. Martel, and S.J. Wind, "Carbon nanotube electronics", Proceedings of the IEEE, vol. 91, issue. 9, pp. 1772-1784, Nov. 2003

[2] S. Chopra, S. Natarajan, and A.M. Rao, "Gas Sensing using Carbon nanotube-based resonator" Proceedings of the IEEE Sensors. pp. 399402, Vienna, Austria, Oct 2004
[3] N. Rouhi, D. Jain, and P.J. Burke, "Nanoscale Devices for Large-Scale Applications", IEEE Microwave Magazine. vol.11, n.7, pp.72-80, Dec. 2010

[4] P. Russer, N. Fichtner, P. Lugli, W. Porod, J.A. Russer, and H. Yordanov, "Nanoelectronics-Based Integrated Antennas", IEEE Microwave Magazine. vol.11, n.7, pp.58-71, Dec. 2010

[5] A. Mehdipour, I. Rosca, A. Sebak, C.W. Trueman, and S.V. Hoa, "Advanced Carbon-Fiber Composite Materials for RFID Tag Antenna Applications" Applied Computational Electromagnetics Society ACES Journal, Special Issue on Computational and Experimental Techniques for RFID Systems and Applications, Part I, pp. 218-229, vol. 25, no. 3, March 2010

[6] Y. Zhou, Y. Bayram, F. Du, L. Dai, and J. Volakis, "Polymer-Carbon Nanotube Sheets for Conformal Load Bearing Antennas" IEEE Transactions on Antennas and Propagation, pp. 2169-2175, vol. 58, no. 7, July 2010

[7] L. Yang, R. Zhang, D. Staiculescu, C.P. Wong, and M.M. Tentzeris, "A Novel Conformal RFID-Enabled Module Utilizing Inkjet-Printed Antennas and Carbon Nanotubes for Gas-Detection Applications" IEEE Antennas and Propagation Letters, vol. 8, pp. 653-656, 2009

[8] A. Mehdipour, I. Rosca, A. Sebak, C. Trueman, and S. Hoa, "FullComposite Fractal Antenna Using Carbon Nanotubes for Multiband Wireless Applications" IEEE Antennas and Wireless Propagation Letters, pp. 891-894, vol. 9, 2010

[9] K.G. Ong, K. Zeng, and C.A. Grimes, "A Wireless, Passive Carbon Nanotube-Based Gas Sensor" IEEE Sensors Journal, pp. 82-88, vol. 2, no. 2, April 2002

[10] P.V. Nikitin, K.V.S. Rao, "Theory and Measurement of Backscattering from RFID Tags", IEEE Antennas Propag. Mag., vol. 48, n. 6, pp. 212218, Dec. 2006.

[11] M. Meyyappan, Carbon Nanotubes Science and Applications, CRC Press LLc, 2005

[12] P.J.F. Harris, Carbon Nanotube Science Synthesis, properties and applications, Cambridge University Press, Cambridge, 2009

[13] G. Marrocco, "The Art of UHF RFID Antenna Design: ImpedanceMatching and Size Reduction Techniques" IEEE Antennas and Propagation Magazine, vol. 50, issue 1, pp. 66-79, Feb 2008

[14] C. Occhiuzzi, and G. Marrocco, "Sensing the Human Body by Implanted Passive RFID Tags" Proceedings of the Fourth European Conference on Antennas and Propagation, pp. 1-5, Barcelona, Spain, 2010 